\title{
INSETOS ASSOCIADOS À GRAVIOLEIRA (Annona muricata L., ANNONACEAE) NÄ REGIÃO DE MANAUS, AMAZONAS, BRASIL
}

\author{
Neusa HAMADA ${ }^{1}$, Ana Lúcia Silva GOMES², Guy COUTURIER ${ }^{3}$, Beatriz \\ RONCHI-TELES ${ }^{1}$
}

RESUMO - Os insetos da graviola (Annonaceae: Annona muricata L.) foram estudados na região de Manaus, Amazonas, Brasil. Encontram-se 37 espécies, entre os quais, seis foram consideradas prejudiciais, quatro atacando o fruto (Bephratelloides pomorum F. (Eurytomidae), Cerconota anonella Sepp (Stenomatidae), Membracis suctifructus Boulard \& Couturier (Membracidae) e Pinnaspis aspidistrae Signoret (Diaspididae); duas nas folhas jovens; (Aphis spiraecola Patch e A. gossypii Glover (Aphididae); e uma perfurando tronco e ramos, Cratosomus bombina (Curculionidae). Trinta e quatro espécies são registradas pela primeira vez.

Palavras-chave: Amazonas, Annona muricata, insetos praga, graviola.

Insects Associated to Soursop (Annona muricata L., Annonaceae) in the Manaus Region, Amazonas, Brazil.

ABSTRACT - Insects collected on soursop (Annonaceae: Annona muricata L.) nearby Manaus, Amazonas, Brazil, are reported. Thirty-seven species were found, but only six were considered injurious to the plant: Bephratelloides pomorum F. (Eurytomidae), Cerconota anonella Sepp (Stenomatidae), Membracis suctifructus Boulard \& Couturier (Membracidade) and Pinnaspis aspidistrae Signoret (Diaspididae) attacking fruits; Aphis spiraecola Patch and Aphis gossypii Glover (Aphididae) feeding on leaves, and Cratosomus bombina F. (Curculionidae) boring trunks and branches. Twenty four species are recorded for the first time.

Key-words: Amazonas, Annona muricata, Annonaceae, Insect pest, soursop.

Agravioleira (Annonaceae: Annona muricata L.) é uma planta originária das Antilhas, estritamente tropical, podendo atingir seis metros de altura, e produzindo frutos que pesam de dois a três quilos (Warumby, 1983). Os frutos são muito apreciados pela população, sendo consumidos ao natural ou utilizados na forma de suco, sorvete e creme. Apesar do seu valor econômico, o seu cultivo, na Amazônia Central, é muito restrito e, em parte, atribuido ao intenso ataque por insetos que essa planta sofre. Embora esses insetos causem grandes problemas, chegando às vezes a destruir uma produção inteira, são raros os trabalhos sobre eles na região.
No Brasil, as espécies Bephratelloides pomorum $\mathrm{F}$. (Eurytomidae), Cerconota anonella Sepp (Stenomatidae) e Cratosomus bombina F. (Curculionidae) são registradas como as mais prejudiciais às gravioleiras (Bondar, 1939, Caloba \& Silva; 1995; Fazolin \& Ledo, 1994; Melo et al., 1983; Ronchi-Teles \& Hamada, 1991; Warumby, 1981). Ferreira Filho et al. (1985) tentaram avaliar se havia diferenças na incidência da broca do tronco da gravioleira (Cratosomus sp.) em dois tipos de consórcios de fruteiras (mono e policultura) na região de Manaus (AM), mas os resultados não indicaram diferenças entre os dois

\footnotetext{
Instituto Nacional de Pesquisas da Amazônia - INPA/CPEN, Caixa Postal 478 69011-970, Manaus, AM.

2 Bolsista - PIBIC/INPA/UA/CNPq.

3 ORSTOM, 213 rue Lafayette, 75480-Paris Cedex 10, França
} 
tratamentos e os autores concluíram que seriam necessários estudos bioecológicos sobre a broca para se entender os fatores que determinam a distribuição desta espécie.

$\mathrm{Na}$ Colômbia e Venezuela os seguintes insetos são reportados como insetos pragas de gravioleiras: Thecla ortignus L. (lagartas atacam as flores) e outras espécies de Lycaenidae que atacam os frutos; C. anonella e Talponia spp. (Grapholitidae) provocando danos semelhantes nos frutos; $C$. bombina, broca do tronco e dos ramos; Bephrata maculicollis Cameron, perfurador da semente; Lonchaea pendula Bezzi (Lonchaeidae), cujas larvas atacam o mesocarpo, o minador de folhas Leucoptera sp.(Lyonetiidae) e o percevejo Corythucha sp. (Tingidae). (Arango, 1975; Araque, 1967; Dominguez Gil, 1977; Gutiérrez \& Tróchez, 1977; Marin Acosta, 1973).

O presente trabalho tem como objetivo contribuir para o conhecimento dos insetos que ocorrem na gravioleira, na região de Manaus, identificando aqueles que the causam maiores danos. A área de estudo abrangeu o Campus do V-8 (INPA), Estação Experimental de Fruticultura Tropical do INPA, localizada na BR 174, km 40; FUCADA, BR 174, km 38; e Fazenda NAF6, BR $174 \mathrm{~km} 14 \mathrm{e}$ em algumas áreas residenciais de Manaus. As coletas de insetos foram realizadas semanalmente no periodo de abril de 1992 a março de 1993, entretanto, observações esporádicas foram feitas desde 1988. A amostragem foi realizada de forma qualitativa, por meio de rede entomológica e catação manual; todas as gravioleiras presentes na área eram examinadas até a altura máxima de $2 \mathrm{~m}$. No laboratório, os imaturos foram acondicionados em frascos de vidro ou em recipientes plásticos, cobertos com um pedaço de filó, preso por ligas de elástico, e alimentados até a obtenção dos adultos ou de possíveis parasitóides. Os espécimes adultos foram montados, etiquetados e identificados até o nivel de familia, e depois enviados para especialistas, para identificação genérica ou específica; e posteriormente todos os espécimens foram depositados na coleção de referência de insetos de importância agrícola da Coleção de Invertebrados do INPA.

Foram coletadas 37 espécies de insetos associadas à gravioleira, sendo 16 de Homoptera, nove de Lepidoptera, cinco de Coleoptera, trếs de Diptera, três de Hemiptera, e uma de Hymenoptera. Os insetos coletados sobre gravioleira, na área de estudo, que puderam ser identificados abaixo do nivel de família, estão relacionados na Tabela 1 . Sete espécies (uma de Coccoidea, uma de Nitidulidae, duas de Limacodidae, uma de Hesperiidae, uma de Noctuidae e uma de Lycaenidae) não foram incluídas nesta tabela devido ao fato de não terem sido identificadas até o nível genérico/especifico.

Os insetos considerados mais prejudiciais foram: a) Cerconota anonella, suas larvas perfuram os frutos, alimentamse da polpa, destruindo-a e inutilizando os frutos para a comercialização; b) Cratosomus bombina, as larvas brocam ramos e troncos (Figs. 1 e 2) de forma descendente, ocasionando redução na produção, sendo que os ramos finos atacados ressecam; c) Bephratelloides pomorum cujas larvas se desenvolvem no interior das sementes (Figs. 3), 


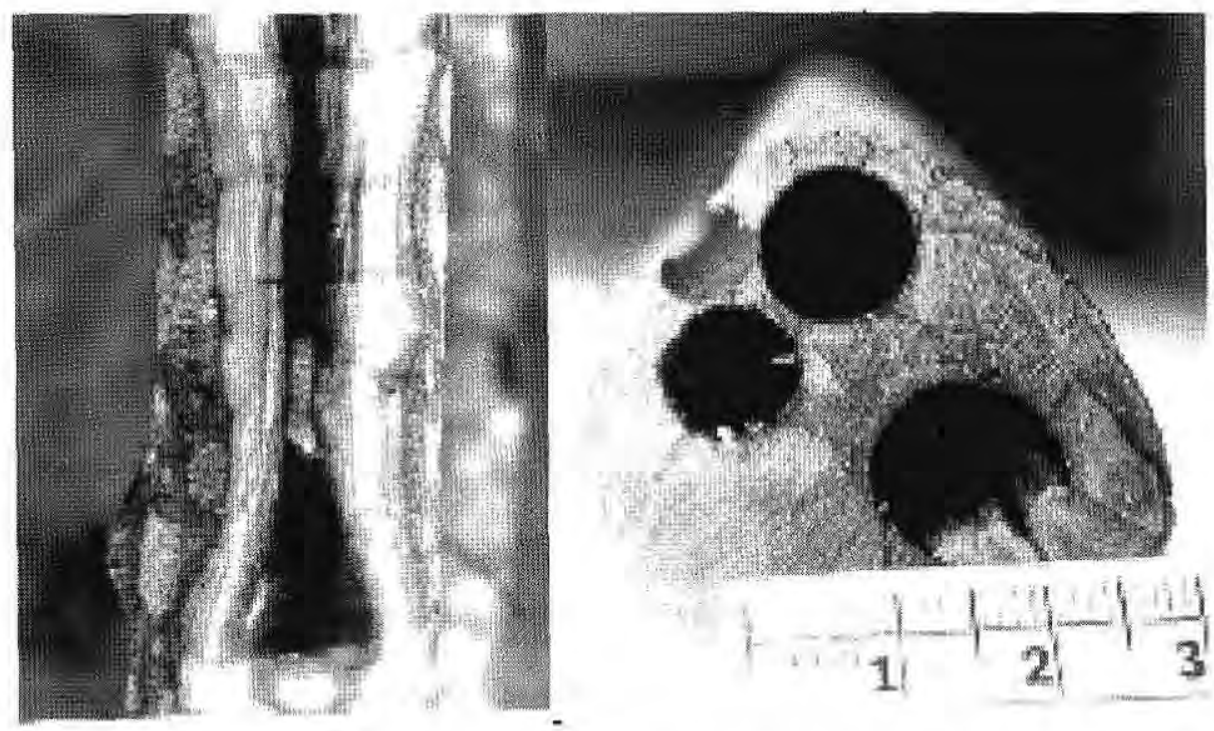

Figura 1. Larya de Cratosomus bombina (Curculionidade) e dano provocado no tronco da gravioleira (Annona muricata).

Figura 2. Galerias formadas pelas larvas de Cratosomus bombina (Curculionidae) em galhos da gravioleira (Annona muricata).
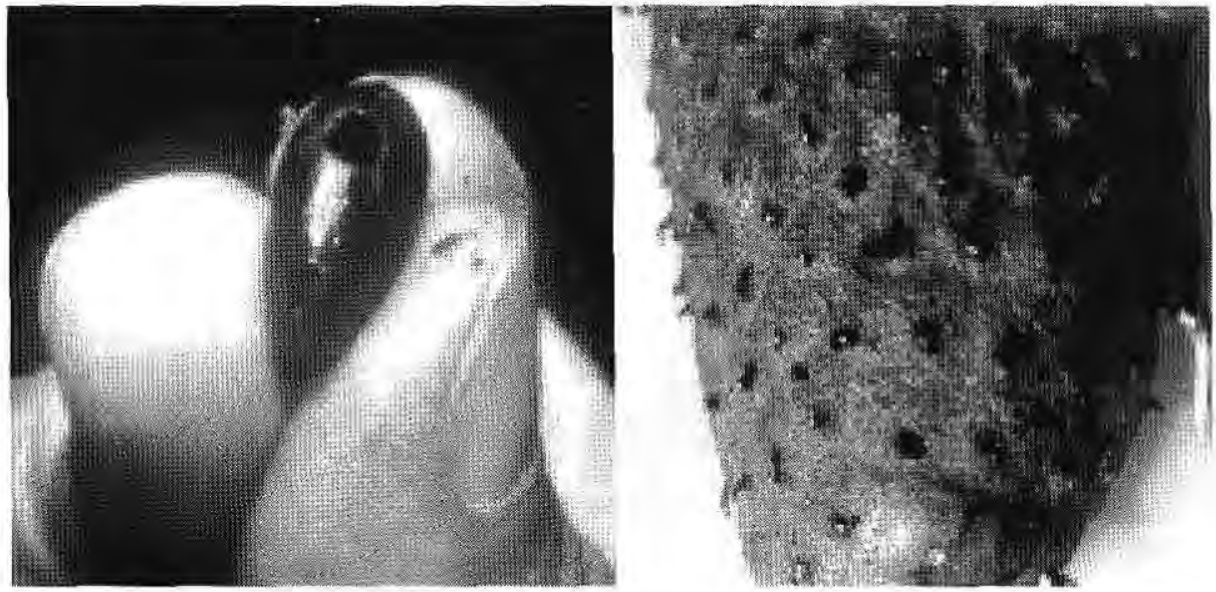

Figura 3. Semente da graviola (Annona muricata) com orificio produzido por Bephratelloides pomorum (Eurytomidae).

Figura 4. Orificios de saida do adulto de Bephratelloides pomorum (Eurytomidae) em graviola (Annona muricata). 
tornando-as improprias para a germinação. Os adultos dessa espécie saem do interior do fruto através de uma perfuração que fazem na casca (Fig. 4), que também facilita a entrada de microorganismos, prejudicando a comercializaçăo do fruto; d) Membracis suctifructus (Membracidae), quando a infestação por adultos e ninfas é alta (Fig.5) os frutos tornam-se deformados e enegrecidos; e) também os pulgões, Aphis gossypii e Aphis spiraecola (Aphididae), em altas infestações, prejudicam as folhas novas, deixando-as deformadas; f) Pinnaspis aspidistrae (Diaspididae) provoca deformações nos frutos (Fig.6), inviabilizando a sua comercialização.

As espécies de Cocytius (C. antaeus e C. duponchel) (Sphingidae) consomem uma grande quantidade de folhas, entretanto, sofrem um intenso controle natural. A maioria dos ovos $(\mathrm{n}=22,81,8 \%)$ coletados no campo, estava parasitada por Telenomus sp. (Scelionidae); as lagartas podem ser parasitadas por Cotesia $\mathrm{sp}$. (Braconidae), e no estágio de pupa, por uma espécie de Tachinidae.

$\mathrm{O}$ número de espécies de insetos associados à gravioleira, na região de Manaus, passa de 11 (Caloba \& Silva, 1995) para 42, incluindo cinco espécies (Antiteuchus minor Engl. (Pentatomidae), Ceroplastes floridensis Coms., Ceroplastes sinensis Del. (Coccidae), Membracis foliata L. (Membracidae) e Campsus argyreus (L.) (Curculionidae) registradas por Caloba \& Silva (1995) que não foram coletadas durante $o$ periodo de estudo. As novas ocorrências constatadas são assinaladas com um asterisco (*) na Tabela 1. As larvas dos dipteros (Neriidae e Lonchaeidae) foram coletadas em frutos em início de decomposição, não sendo portanto, prejudiciais à produção comercial da graviola. As três espécies que causam maior dano à planta $(C$. anonella, $C$. bombina e $B$. pomorum) na região de

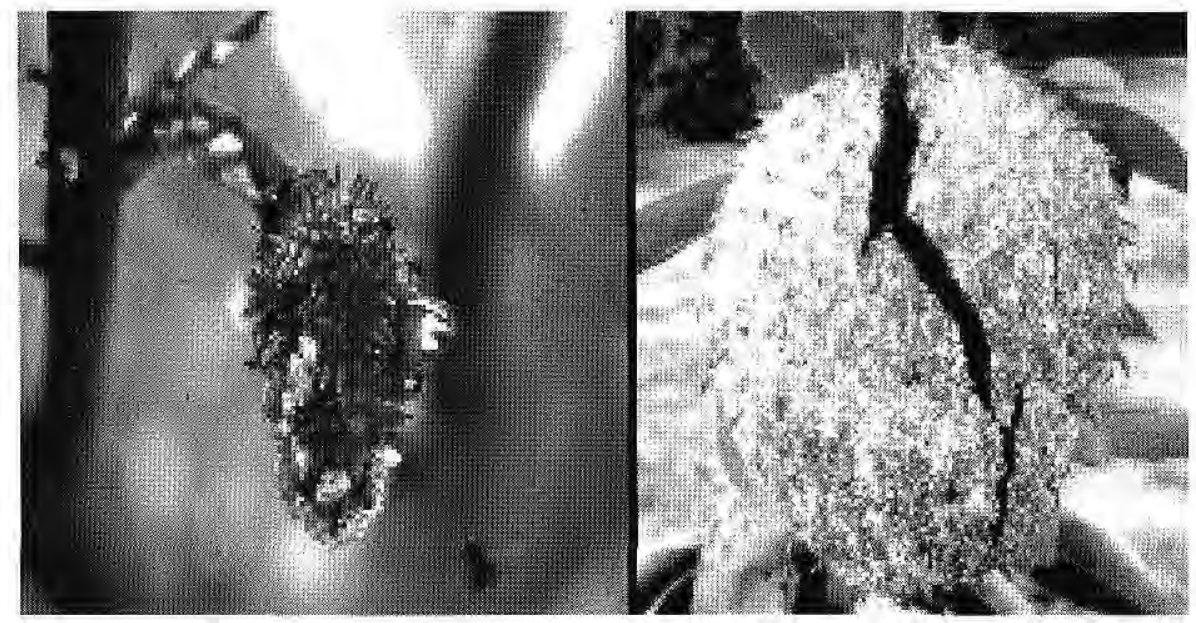

Figura 5. Adulto e ninfas de Membracis suctifructus (Membracidae) e dano provocado sobre o fruto jovem da gravioleira (Annona muricala).

Figura 6. Pinnaspis aspidistrae (Diaspididae) e dano provocado em graviola (Annona muricata). 
Tabela 1. Insetos da gravioleira (Annonaceae: Annona muricata Linn.) coletados na região de Manaus (AM), no periodo de abril/1992 A Fevereiro/1993.

NOME CIENTIFICO

HEMIPTERA

*Acanthocephala parensis Dallas

"Antiteuchus sepulchralis F.

*Teleonemia morio Stoll

HOMOPTERA

Aleurodicus sp.

"Aphis gossypii Glover

Aphis spiraecola Patch

*Membracis suctifructus

Boulard \& Couturier

${ }^{*}$ Membracis tectigera Olivier

"Enchenopa albidorsa Fairm.

"Guayaquila tenuicornis Walker

*Amastris obtegens Fabricius

*Ceresa peruensis Remes Lenicov

"Tunelia longula Burm.

*Stegaspis fronditia L.

"Cyphonia clavata Fabricius

"Enchophyllum sp.

"Bocydium globulare F.

*Pinaspis aspidistrae Signoret

\section{COLEOPTERA}

Cratosomus bombina $\mathrm{F}$.

*Homophoeta aequinoctialis L.

-Pleurica aemula Weise

-Diabrotica sp.

DIPTERA

*Neosilba sp.

"Nerius pilifer $\mathrm{F}$.

- Olyphidops carrerai Aczel

\section{LEPIDOPTERA}

Cerconota anonelia Sepp

Cocytius antaeus Stoll

- Cocytius duponchel Pory

- Gonodonta fulvangulata Geyer

HYMENOPTERA

Bephratelloides pomorum $\mathrm{F}$.
(COREIDAE)

(PENTATOMIDAE)

(TINGIDAE)

(ALEYRODIDAE)

(APHIDIDAE)

(APHIDIDAE)

(MEMBRACIDAE)

(MEMBRACIDAE)

(MEMBRACIDAE)

(MEMBRACIDAE)

(MEMBRACIDAE)

(MEMBRACIDAE)

(MEMBRACIDAE)

(MEMBRACIDAE)

(MEMBRACIDAE)

(MEMBRACIDAE)

(MEMBRACIDAE)

(DIASPIDIDAE)

(CURCULIONIDAE)

(CHRYSOMELIDAE)

(CHRYSOMELIDAE)

(CHRYSOMELIDAE)

(LONCHAEIDAE)

(NERIIDAE)

(NERIIDAE)

(STENOMATIDAE)

(SPHINGIDAE)

(SPHINGIDAE)

(NOCTUIDAE)

(EURYTOMIDAE)
Partes da planta atacada

Folhas e trutos

Folhas

Folhas e frutos

Folhas

Folhas e flores

Folhas e flores

Folhas, ramos e frutos

Folhas e ramos

Folhas e ramos

Folhas e ramos

Folhas e ramos

Folhas e ramos

Folhas e ramos

Folhas e ramos

Folhas e ramos

Folhas e ramos

Folhas e ramos

Ramos e frutos

Ramos e tronco

Folhas

Folhas

Folhas e frutos

Frutos

Frutos

Frutos

Frutos

Folhas

Folhas

Folhas

Sementes

Nota: ${ }^{*}$ = nova ocorrência em gravioleira na regiăo de Manaus 
Manaus, são as mesmas de importância econômica nas diversas áreas de cultivo dessa frutífera na América do Sul (Bondar, 1939; Caloba \& Silva, 1995; Fazolin \& Ledo, 1994; Melo et al, 1983; Ronchi-Teles \& Hamada, 1991; Warumby, 1981; Arango, 1975; Araque, 1967; Dominguez Gil, 1978; Gutiérrez \& Tróchez, 1977 e Marin Acosta, 1973). Entretanto, a maioria das outras espécies é reportada pela primeira vez nesta frutífera na Amazônia Central (Tab. 1).

Fatores bióticos e abióticos podem afetar a entomofauna de diferentes culturas, induzindo $o$ aumento populacional de uma determinada espécie anteriormente em equilíbrio, elevando-a à condição de praga. Dessa forma, estudos bioecológicos (envolvendo parasitóides, predadores e doenças) dos insetos associados à gravioleira, especialmente $C$. bombina, $B$. pomorum, C. anonella, A. spiraecola, A. gossypii e $P$. aspidistrae, considerados os mais prejudiciais, são imprescindiveis para se determinar métodos racionais de controle populacional, de modo que essa cultura passe do status de cultivo de fundo de quintal para ser desenvolvida em escala comercial na região.

\section{AGRADECIMENTOS}

Os autores manifestam agradecimentos às seguintes pessoas pela identificação dos insetos: J.Y. Rasplus; (Eurytomidae), G.H. Rosado-Neto (Curculionidae), A.M. Sakakibara (Homoptera), C. Mota (Sphingidae), D. Matile-Ferrero (Diaspididae); P.M. Marsh (Braconidae); L. Albuquerque
(Neriidae); G. Ramaudiere (Aphididae) e N. Berti (Chysomelidae).

\section{Bibliografia citada}

Arango, F.T. 1975. La Guanabana (Annona muricata L.). Revta Esso Agricola, 21 (2): 9-10.

Araque, R. 1967. La Guanabana. Consejo de bienestar rural, Serie de cultivos n. 13, Caracas, Venezuela, 16p.

Bondar, G. 1939. Sobre alguns insetos nocivos às anonáceas frutíferas na Bahia e descrição de um novo curculionideo. A Bahia Rural, 6: 65-66.

Caloba, J.; Silva, N. M. 1995. Insetos associados a graviola Annona muricata L. e biriba, Rollinia mucosa (Jacq.) Bail no Estado do Amazonas. An, Soc, Entomol. Brasil. 24(1): 179-182.

Domingues Gil, O. E. 1978. Insectos perjudiciales del guanábano (Annona muricata L.) en el Estado Zulia, Venezuela. Rev. Facult. de Agronomia.3(4): 149-163p.

Fazolin, M.; Ledo A. da S. 1994. Entomofauna relacionada com os frutos de graviola no dois primeiro anos da produção, em Rio Branco, Acre. Vol. 2, p. 587 Anais Congresso Brasileiro de Fruticultura, 13, Salvador, $1211 \mathrm{p}$.

Ferreira Filho, W.C.; Flores, W.B.C; Clemente, C.R.; Benson, W.W. 1985. A influência de dois tipos de consórcios de fruteiras na incidência de broca (Cratosomus sp.) na graviola (Annona muricata L.). Acta Amazonica, 15 (1-2): 3-11p.

Gutiérrez, B.A.; Trochez, A. 1977. Estudio sobre plagas de las Anonaceas en el Valle del Cauca. Rev. Col de Entomologia, 3: 1-2.

Marin Acosta, J.C. 1973. Lista preliminar de plagas de Annonacea, Nispero (Achras zapota L.) y Guayaba (Psidium guajava L.) en Venezuela. Agronomia Tropical, 23: 205216.

Melo, G.S.; Gonzaga Neto, L.; Moura, R.J.M. 1983. Cultivo da Gravioleira. (Annona muricata L.). Instruções técnicas do IPA, Recife, PE, 3p. 
Ronchi-Teles, B.; Hamada, N. 1991. Estudos bioecológicos de insetos prejudiciais à agricultura como subsidio para o manejo de pragas na Amazônia in Val, A., Figliuolo, R.; Feldberg, E.(Eds.) Bases Cientificas para Estratégias de Desenvolvimento da Amazônia: Fatos e Perspectiva Vol. 1. Instituto Nacional de Pesquisas da Amazônia (INPA). Manaus, Amazonas. p. 233-236.

Warumby, J.F. 1981. Pragas de gravioleira. Correio Agricola. 3: 360. 